\title{
The Wilson-Bappu effect: A tool to determine stellar distances ${ }^{\star}$
}

\author{
G. Pace ${ }^{1,2}$, L. Pasquini ${ }^{2}$, and S. Ortolani ${ }^{3}$ \\ 1 Dipartimento di Astronomia, Università di Trieste, via G. B. Tiepolo 11, 34131 Trieste, Italy \\ 2 European Southern Observatory, Karl Schwarzschild Strasse 2, 85748 Garching bei München, Germany \\ 3 Dipartimento di Astronomia, Università di Padova, Vicolo dell’Osservatorio 5, 35122 Padova, Italy
}

Received 12 July 2002 / Accepted 30 January 2003

\begin{abstract}
Wilson \& Bappu (1957) have shown the existence of a remarkable correlation between the width of the emission in the core of the $\mathrm{K}$ line of $\mathrm{CaII}$ and the absolute visual magnitude of late-type stars.

Here we present a new calibration of the Wilson-Bappu effect based on a sample of 119 nearby stars. We use, for the first time, width measurements based on high resolution and high signal to noise ratio CCD spectra and absolute visual magnitudes from the Hipparcos database.

Our primary goal is to investigate the possibility of using the Wilson-Bappu effect to determine accurate distances to single stars and groups.

The result of our calibration fitting of the Wilson-Bappu relationship is $M_{V}=33.2-18.0 \log W_{0}$, and the determination seems free of systematic effects. The root mean square error of the fitting is $0.6 \mathrm{mag}$. This error is mostly accounted for by measurement errors and intrinsic variability of $W_{0}$, but in addition a possible dependence on the metallicity is found, which becomes clearly noticeable for metallicities below $[\mathrm{Fe} / \mathrm{H}] \sim-0.4$. This detection is possible because in our sample $[\mathrm{Fe} / \mathrm{H}]$ ranges from -1.5 to 0.4 .

The Wilson-Bappu effect can be used confidently for all metallicities not lower than $\sim-0.4$, including the LMC. While it does not provide accurate distances to single stars, it is a useful tool to determine accurate distances to clusters and aggregates, where a sufficient number of stars can be observed.

We apply the Wilson-Bappu effect to published data of the open cluster M67; the retrieved distance modulus is of 9.65 mag, in very good agreement with the best distance estimations for this cluster, based on main sequence fitting.
\end{abstract}

Key words. stars: distances - stars: late-type - line: profiles

\section{Introduction}

The $\mathrm{K}$ line of $\mathrm{CaII}$ is the deepest and widest absorption line in the spectra of the late type stars, and its core shows the characteristic double reversal profile: an emission with a central selfabsorption (see e.g. Pasquini et al. 1988).

Since the discovery by Wilson \& Bappu (1957) of the existence of a linear relationship between the logarithm of the width of the Ca II emission $\left(W_{0}\right)$ and the stellar absolute visual magnitude (the so called Wilson-Bappu effect), several calibrations of this effect have been attempted. However the Wilson-Bappu relationship (WBR) has only seldom been used to determine stellar distances to single stars or aggregates.

The reliability of past calibrations of the WBR has been limited by the lack of two crucial elements:

1. Precise and accurate parallaxes for nearby stars;

2. High quality data and objective method of measuring $W_{0}$.

The first point has been overcome by Wallerstein et al. (1999), who produced a new calibration by exploiting the Hipparcos

Send offprint requests to: G. Pace, e-mail: gpace@eso.org

* Observations collected at ESO, La Silla. database. This work however was hampered by the fact that it was still based on $W_{0}$ measurements provided by Wilson (1967, 1976), performed on photographic plates. For our calibration we have used $W_{0}$ measurements performed on new, high quality CCD spectra, so that also the second point has been, for the first time, properly accounted for.

A new, reliable WBR determination is especially interesting since new detectors and state of the art spectrographs can now produce excellent $\mathrm{Ca}$ II data even for stars in stellar clusters and associations as distant as several kiloparsecs. For these clusters and associations, we could therefore apply the WBR to derive their distance. This possible application is the main ground of our effort to retrieve a reliable calibration for the WBR.

\section{Data sample and observations}

The full sample for which spectra have been collected (data shown in Table 1) consists of 152 stars, but the present study is limited to stars with relative parallax errors smaller than $10 \%$. We have also excluded from the original sample 
known multiple systems. After this trimming, the final sample includes 119 stars.

All the stars but the Sun are included in the Hipparcos catalogue, from which trigonometric parallaxes and visual magnitudes have been taken.

The absolute visual magnitude of the Sun has been taken from Hayes (1985).

The sample has been selected in order to span a wide range of luminosities (from $M_{V} \simeq-5$ to $M_{V} \simeq 9$ ).

The observations were obtained between November 1988 and September 1996, at ESO, La Silla, with the Coudé Echelle Spectrometer, at the focus of the Coudé Auxiliary Telescope. The resolution is $R=60000$ and the $S / N$ ratio ranges from $\simeq 30$ to $\simeq 100$ at the bottom of the line (for more details about the first spectra see Pasquini 1992).

For 230 stars, multiple spectra were taken, and for the Sun 9 spectra are available.

Spectral types and metallicities were obtained from the Cayrel de Strobel et al. (1997) catalogue. For the stars not included in this catalogue, spectral types are taken from the Hipparcos database. The projected rotation velocities are from Glẹbocki et al. (2000).

\section{Measurements and calibration}

$W_{0}$ was measured manually on all of the spectra. We have also developed an IDL macro which performs a multi Gaussian fitting to the $\mathrm{K}$-line double-reversal profile, but it has not been used for the final computation because it has proven quite fragile for the correct determination of the broad line absorption profile. We are working on improving the method, and we may report further progress elsewhere.

$W_{0}$ has been computed as the difference in wavelength between the two points taken at the intensity equal to the average between those of the $\mathrm{K} 1$ minimum and $\mathrm{K} 2$ peak on either side of the emission profile (see Fig. 2).

We found that the definition of $W_{0}$ which we have adopted correlates better with $M_{V}$ than two other widths also measured:

- the difference in wavelength between K1 minima;

- the width taken at the intensity equal to the average between those of the $\mathrm{K} 1$ minima and $\mathrm{K} 2$ maxima.

No correction for instrumental broadening was applied to $W_{0}$. The reason is explained in Sect. 5.4.

We notice that our definition of $W_{0}$ differs slightly from others used in the literature. Wilson \& Bappu (1957) define $W$ as the difference in wavelength between the red edge and the violet edge of the emission profile. They apply to the measured value in the spectra a linear correction: $W_{0}=W-15 \mathrm{~km} \mathrm{~s}^{-1}$. Wilson (1959) kept the original definition but applied a revised correction: $18 \mathrm{~km} \mathrm{~s}^{-1}$ instead of 15 . Lutz (1970) introduced a new definition of $W_{0}$, defining it as the width at half of the maximum of the emission profile. Lutz's definition is very similar to ours with the exception that we found the our definition easier to use in case of difficult spectra and more robust (see below).

$W_{0}$ measurements are subject, of course, to measurement errors. For each star we have computed an accuracy qualifier, $\Delta W_{0}$, in the following way. For the stars with two or more spectra available we derived it as the half of the difference between the largest and smallest measured widths. For stars with only one available spectrum, we measured $W_{0}$ with multiple methods, and took the difference between the extrema of the measurements. Then we added quadratically to this value (which in some cases was 0 ) that of the Sun $(0.030 \AA$ ), whose $W_{0}$ measurements variations are supposed to be caused only by the intrinsic variation of the line width and by the limit of the resolution power of the spectrograph. We have used $\Delta W_{0}$ as an estimate of the mean error in $W_{0}$. The standard error of $\log W_{0}$ (where $W_{0}$ is in $\mathrm{km} \mathrm{s}^{-1}$ ) for each star, is retrieved applying the propagation of the mean error.

Independent of the signal to noise ratio, for some stars it is intrinsically more difficult to measure $W_{0}$. This is because their spectra show asymmetric self absorption, either produced by interstellar lines or by blueshifted winds or cosmic rays. Inactive, low luminosity stars will typically show shallow reversals, which are more difficult to measure.

Some of the most doubtful examples and difficult cases are presented in Fig. 1, in order to make the reader acquainted with the spectra and the possible error sources. In some cases, when strong blending was present or the profile was highly asymmetric, we have measured $W_{0}$ by doubling the value measured for the "clean" half of the line. Anyway, the majority of the spectra we dealt with were as good as the one showed in Fig. 2.

We have also computed the standard error on the absolute magnitude of each star. This error has two components: the mean error on the apparent visual magnitude (which is, in most of the cases, negligible) and the error given by the uncertainty in the parallax, which has to be computed via the propagation of the error.

The fit of the WBR was performed by means of the IDL routine "fitexy", which implements the algorithm described in Press et al. (1989). The algorithm fits a straight line to a set of data points by taking into accounts errors on both coordinates.

We repeated the solution by rejecting stars not passing $3 \sigma-$, $2.5 \sigma-$ or $2 \sigma-$ criteria. The results so obtained are presented in Table 2.

It is fundamental to note that, independent of the different sigma clipping criterion used, the solutions found are extremely stable, giving the same fit to within $1 \sigma$.

We adopt in the following: $M_{V}=33.2-18.0 \cdot \log W_{0}\left(W_{0}\right.$ is in $\mathrm{km} \mathrm{s}^{-1}$ ) obtained rejecting HD 63077 and HD 211998, with a standard deviation: $\sigma_{\mathrm{WBR}}=0.6 \mathrm{mag}$. The two rejected stars are the most metal poor of the sample, which we will argue in Sect. 5.5 is the most likely cause of their large residuals.

Hereafter we indicate with $M_{V}(K)$ the value of $M_{V}$ derived for a single star from its $W_{0}$ via the WBR. In Fig. 3 the $\log W_{0}$ vs. $M_{V}$ diagram is shown, with the error bars representing standard errors in both coordinates. The calibration line retrieved is also plotted.

\section{Comparison with other results}

In order to evaluate the external robustness of the relationship, a comparison with other independent investigations is fundamental. Many attempts in the past 40 years have been 
Table 1. Our data sample. The full sample consists of 152 stars, 33 of which have not been used in the present study, because of their high measurement error in the parallax $\left(\frac{\sigma_{\pi}}{\pi}>0.1\right)$ or because they are multiple systems. We have flagged their HD identificators with an asterisk. Column 1: HD ID number of the star. Column 2: $W_{0}$ in $\AA$. Column 3: accuracy qualifier for $W_{0}$, again in $\AA$. Its mean value is 0.036 . Column 4: absolute visual magnitude using Hipparcos parallaxes. For the Sun we used the value given by Hayes (1985). Column 5: spectral type from Cayrel De Strobel Catalogue. When the spectral type is not available from this catalogue the data is taken from the Hipparcos database, and is flagged with a dagger. Column 6: mean metallicity, when available from Cayrel De Strobel Catalogue. Column 7: projected rotation velocity, when available from Glẹbocki et al. (2000).

\begin{tabular}{|c|c|c|c|c|c|c|}
\hline Star & $W_{0}[\AA]$ & $\Delta W_{0}[\AA]$ & $M_{V}$ & Sp.Type & {$[\mathrm{Fe} / \mathrm{H}]$} & $V \cdot \sin i$ \\
\hline SUN & 0.49 & 0.030 & 4.82 & $\mathrm{G} 2 \mathrm{~V}$ & 0.00 & 1.6 \\
\hline HD 203244 & 0.47 & 0.030 & 5.42 & G5V & -0.21 & - \\
\hline HD 17051 & 0.62 & 0.030 & 4.22 & G0V & -0.04 & 5.7 \\
\hline HD $1273^{*}$ & 0.5 & 0.030 & 5.03 & $\mathrm{G} 2 \mathrm{~V} \dagger$ & -0.61 & - \\
\hline HD 20407 & 0.41 & 0.042 & 4.82 & G1V & -0.55 & - \\
\hline HD 20766 & 0.50 & 0.030 & 5.11 & $\mathrm{G} 2.5 \mathrm{~V}$ & -0.25 & - \\
\hline HD 20630 & 0.53 & 0.030 & 5.03 & G5Vvar & 0.11 & 4.6 \\
\hline HD 20807 & 0.44 & 0.036 & 4.83 & G1V & -0.21 & - \\
\hline HD 20794 & 0.43 & 0.030 & 5.35 & G8V & -0.38 & - \\
\hline HD 26491 & 0.50 & 0.058 & 4.54 & G3V & -0.23 & - \\
\hline HD 1581 & 0.49 & 0.15 & 4.56 & F9V & -0.20 & 3 \\
\hline HD 30495 & 0.55 & 0.030 & 4.87 & G3V & 0.11 & 3 \\
\hline HD 32778 & 0.42 & 0.030 & 5.28 & G0V & -0.61 & - \\
\hline HD 34721 & 0.60 & 0.032 & 3.98 & G0V & -0.25 & - \\
\hline HD 36435 & 0.48 & 0.030 & 5.53 & G6-G8V & -0.02 & 4.5 \\
\hline HD 39587 & 0.56 & 0.030 & 4.70 & G0V & 0.08 & 9.3 \\
\hline HD 43834 & 0.51 & 0.036 & 5.05 & G6V & 0.01 & 1.8 \\
\hline HD 48938 & 0.53 & 0.042 & 4.31 & $\mathrm{G} 2 \mathrm{~V}$ & -0.47 & - \\
\hline HD 3443 & 0.47 & 0.036 & 4.61 & $\mathrm{~K} 1 \mathrm{~V}$ & -0.16 & 2.7 \\
\hline HD 53705 & 0.60 & 0.036 & 4.51 & G3V & -0.30 & - \\
\hline HD 3795 & 0.46 & 0.030 & 3.86 & G3-G5V & -0.73 & - \\
\hline HD 63077 & 0.41 & 0.032 & 4.45 & G0V & -0.90 & - \\
\hline HD 3823 & 0.60 & 0.042 & 3.86 & G1V & -0.35 & 3 \\
\hline HD $64096^{*}$ & 0.50 & 0.030 & 4.05 & $\mathrm{G} 2 \mathrm{~V} \dagger$ & - & - \\
\hline HD 65907 & 0.52 & 0.050 & 4.54 & G0V & -0.36 & - \\
\hline HD 67458 & 0.47 & 0.036 & 4.76 & G4IV-V & -0.24 & - \\
\hline HD 74772 & 0.89 & 0.032 & -0.17 & G5III & -0.03 & 5.8 \\
\hline HD 202457 & 0.61 & 0.036 & 4.13 & G5V & -0.14 & - \\
\hline HD 202560 & 0.35 & 0.030 & 8.71 & $\mathrm{M} 1-\mathrm{M} 2 \mathrm{~V} \dagger$ & - & - \\
\hline HD 202628 & 0.55 & 0.030 & 4.87 & $\mathrm{G} 2 \mathrm{~V}$ & -0.14 & - \\
\hline HD 202940* & 0.6 & 0.030 & 5.20 & G5V & -0.38 & 1.2 \\
\hline HD 211415 & 0.48 & 0.032 & 4.69 & G3V & -0.36 & 1.7 \\
\hline HD 211998 & 0.47 & 0.036 & 2.98 & A3V & -1.50 & - \\
\hline HD 212330 & 0.51 & 0.067 & 3.75 & G3IV & 0.14 & 1.8 \\
\hline HD 212698 & 0.54 & 0.030 & 4.04 & G3V & 0.08 & 9.7 \\
\hline HD 14412 & 0.40 & 0.042 & 5.81 & G5V & -0.53 & - \\
\hline HD 14802 & 0.62 & 0.032 & 3.48 & $\mathrm{G} 2 \mathrm{~V}$ & 0.10 & 3 \\
\hline HD 104304 & 0.55 & 0.036 & 4.99 & G9IV & 0.17 & 1.7 \\
\hline HD 114613 & 0.56 & 0.030 & 3.29 & $\mathrm{G} 3 \mathrm{~V} \dagger$ & - & 2.7 \\
\hline HD 11695 & 1.02 & 0.030 & -0.57 & $\mathrm{M} 4 \mathrm{III} \dagger$ & - & - \\
\hline HD 194640 & 0.46 & 0.032 & 5.17 & $\mathrm{G} 6-\mathrm{G} 8 \mathrm{~V}+$ & - & - \\
\hline HD $20610^{*}$ & 0.86 & 0.032 & 0.39 & K0III & -0.07 & - \\
\hline HD 209100 & 0.39 & 0.030 & 6.89 & $\mathrm{~K} 4.5 \mathrm{~V}$ & 0.14 & 0.7 \\
\hline HD 211038 & 0.56 & 0.032 & 3.64 & $\mathrm{~K} 0-\mathrm{K} 1 \mathrm{~V}$ & - & - \\
\hline HD 219215 & 1.03 & 0.030 & 0.05 & $\mathrm{M} 2 \mathrm{III} \dagger$ & - & - \\
\hline HD $29503^{*}$ & 0.80 & 0.030 & 1.23 & K0III & -0.11 & - \\
\hline HD 35162 & 0.86 & 0.042 & 0.28 & G8-K0II-III & -0.31 & - \\
\hline
\end{tabular}


Table 1. continued.

\begin{tabular}{|c|c|c|c|c|c|c|}
\hline Star & $W_{0}[\AA]$ & $\Delta W_{0}[\AA]$ & $M_{V}$ & Sp.Type & {$[\mathrm{Fe} / \mathrm{H}]$} & $V \cdot \sin i$ \\
\hline HD 36079* & 0.92 & 0.030 & -0.63 & G5II & -0.20 & 5.1 \\
\hline HD 4128 & 0.94 & 0.032 & -0.30 & KOIII & -0.01 & 3.3 \\
\hline HD 43455 & 1.05 & 0.032 & -1.55 & $\mathrm{M} 2.5 \mathrm{III} \dagger$ & - & - \\
\hline HD 4398 & 0.82 & 0.032 & 0.44 & G8-K0III † & - & - \\
\hline HD 102212 & 1.01 & 0.067 & -0.87 & $\mathrm{MOIII} \dagger$ & - & - \\
\hline HD 111028 & 0.72 & 0.036 & 2.40 & K1III-IV & -0.40 & 1.5 \\
\hline HD 112300 & 1.04 & 0.030 & -0.57 & M3III & -0.09 & - \\
\hline HD 113226 & 0.94 & 0.030 & 0.37 & G8IIIvar & 0.04 & 2.8 \\
\hline HD 114038 & 0.89 & 0.030 & 0.29 & K1III & -0.04 & - \\
\hline HD 115202 & 0.66 & 0.030 & 2.26 & $\mathrm{~K} 1 \mathrm{III} \dagger$ & - & - \\
\hline HD 115659 & 0.92 & 0.030 & -0.04 & G8III & -0.03 & 4.2 \\
\hline HD 117818 & 0.81 & 0.032 & 0.67 & K0III & -0.40 & - \\
\hline HD $119149 *$ & 1.15 & 0.030 & -0.70 & $\mathrm{M} 2 \mathrm{III} \dagger$ & - & - \\
\hline HD 120477 & 0.90 & 0.032 & -0.33 & K5.5IIIvar & -0.23 & 2.2 \\
\hline HD 121299 & 0.83 & 0.032 & 0.70 & K2III & -0.03 & - \\
\hline HD 123123 & 0.83 & 0.030 & 0.79 & $\mathrm{~K} 2 \mathrm{III} \dagger$ & -0.05 & - \\
\hline HD 124294 & 0.95 & 0.030 & 0.00 & $\mathrm{~K} 2.5 \mathrm{IIIb}$ & -0.45 & - \\
\hline HD 125454 & 0.82 & 0.030 & 0.52 & G8III & -0.22 & - \\
\hline HD 126868 & 0.77 & 0.030 & 1.72 & $\mathrm{G} 2 \mathrm{III} \dagger$ & - & 14.3 \\
\hline HD $129312 *$ & 1.04 & 0.030 & -1.37 & G7IIvar & -0.30 & 6.5 \\
\hline HD 130952 & 0.88 & 0.030 & 0.82 & G8III & -0.29 & - \\
\hline HD 133165 & 0.83 & 0.032 & 0.64 & K0.5IIIb & -0.22 & - \\
\hline HD 136514 & 0.85 & 0.030 & 0.98 & K3IIIvar & -0.14 & 0.6 \\
\hline HD 138716 & 0.68 & 0.032 & 2.30 & K1IV & -0.13 & 2.5 \\
\hline HD 140573 & 0.88 & 0.030 & 0.87 & $\mathrm{~K} 2 \mathrm{IIIb}$ & 0.14 & 1.4 \\
\hline HD 141680 & 0.82 & 0.032 & 0.68 & G8III & -0.28 & 1.1 \\
\hline HD $145001 *$ & 0.94 & 0.050 & -0.37 & G8III & -0.26 & 9.9 \\
\hline HD $145206^{*}$ & 1.00 & 0.032 & -0.51 & K4III & 0.04 & 3.2 \\
\hline HD 146051 & 1.12 & 0.030 & -0.85 & M0.5III & 0.32 & - \\
\hline HD 146791 & 0.83 & 0.030 & 0.64 & G9.5IIIb & -0.13 & - \\
\hline HD $148349^{*}$ & 0.90 & 0.030 & -0.71 & $\mathrm{M} 2 \dagger$ & - & - \\
\hline HD $148513^{*}$ & 1.10 & 0.036 & -0.15 & K4III & -0.14 & 0.6 \\
\hline HD $150416^{*}$ & 1.04 & 0.030 & -0.48 & G8II-III & +0.04 & - \\
\hline HD 151217 & 1.00 & 0.032 & 0.00 & K5IIIvar & -0.11 & 2.3 \\
\hline HD 152334 & 0.98 & 0.030 & 0.30 & $\mathrm{~K} 4 \mathrm{III} \dagger$ & - & - \\
\hline HD 152601 & 0.82 & 0.030 & 0.82 & K2III & 0.00 & - \\
\hline HD 161096 & 0.91 & 0.030 & 0.76 & K2III & 0.05 & 2.7 \\
\hline HD $164349 *$ & 1.17 & 0.030 & -1.84 & K0.5IIb & -0.32 & - \\
\hline HD 165760 & 0.91 & 0.095 & 0.33 & G8III & -0.15 & 2.2 \\
\hline HD 168723 & 0.67 & 0.030 & 1.84 & K0III-IV & -0.10 & 2.6 \\
\hline HD $169156^{*}$ & 0.77 & 0.030 & 0.82 & G9IIIb & -0.17 & - \\
\hline HD 169767 & 0.74 & 0.030 & 1.15 & G8-K0III † & - & - \\
\hline HD 170493 & 0.44 & 0.030 & 6.67 & $\mathrm{~K} 3 \mathrm{~V} \dagger$ & - & 3.5 \\
\hline HD 171443 & 0.98 & 0.030 & 0.21 & K3III & 0.09 & 1.8 \\
\hline HD $171967 *$ & 1.10 & 0.032 & -1.57 & $\mathrm{M} 2 \mathrm{III} \dagger$ & - & - \\
\hline HD $173009^{*}$ & 1.11 & 0.032 & -1.14 & G8IIb & 0.05 & 6.0 \\
\hline HD $173764 *$ & 1.80 & 0.036 & -2.40 & G4IIa & -0.15 & 6.5 \\
\hline HD $175775^{*}$ & 1.07 & 0.030 & -1.76 & G8-K0II-III & -0.19 & - \\
\hline HD 176678 & 0.84 & 0.032 & 0.73 & K1IIIvar & -0.19 & - \\
\hline HD 177565 & 0.51 & 0.030 & 4.98 & G5IV & 0.03 & - \\
\hline HD 17970 & 0.44 & 0.050 & 6.01 & $\mathrm{~K} 1 \mathrm{~V} \dagger$ & - & - \\
\hline HD $181391 *$ & 0.77 & 0.030 & 1.61 & G8III & -0.21 & 2.8 \\
\hline HD 182572 & 0.60 & 0.032 & 4.27 & G8IV & 0.38 & 2.3 \\
\hline HD $183630 *$ & 1.02 & 0.050 & -0.90 & M1IIvar $\dagger$ & - & - \\
\hline
\end{tabular}


Table 1. continued.

\begin{tabular}{|c|c|c|c|c|c|c|}
\hline Star & $W_{0}[\AA]$ & $\Delta W_{0}[\AA]$ & $M_{V}$ & Sp.Type & {$[\mathrm{Fe} / \mathrm{H}]$} & $V \cdot \sin i$ \\
\hline HD 184406 & 0.79 & 0.030 & 1.80 & K3IIIb & 0.05 & 1.3 \\
\hline HD $186791^{*}$ & 1.32 & 0.030 & -3.02 & K3II & 0.00 & 3.5 \\
\hline HD 188310 & 0.91 & 0.032 & 0.73 & G9IIIb & -0.32 & 2 \\
\hline HD 189319 & 1.13 & 0.030 & -1.11 & $\mathrm{~K} 5 \mathrm{III} \dagger$ & - & - \\
\hline HD 190248 & 0.54 & 0.030 & 4.62 & G7IV & -0.26 & - \\
\hline HD 190406 & 0.49 & 0.030 & 4.56 & $\mathrm{G} 1 \mathrm{~V} \dagger$ & - & 5 \\
\hline HD 191408 & 0.38 & 0.030 & 6.41 & $\mathrm{~K} 2 \mathrm{~V} \dagger$ & - & 0.1 \\
\hline HD 194013 & 0.75 & 0.032 & 0.91 & G8III-IV & -0.03 & 1 \\
\hline HD 195135 & 0.88 & 0.036 & 1.07 & K2III & 0.03 & - \\
\hline HD 196574* & 0.86 & 0.030 & -1.04 & G8III & -0.13 & 3.7 \\
\hline HD 196758 & 0.89 & 0.036 & 0.77 & K1III & -0.12 & 1.8 \\
\hline HD 196761 & 0.42 & 0.030 & 5.53 & $\mathrm{G} 8-\mathrm{K} 0 \mathrm{~V} \dagger$ & - & - \\
\hline HD 198026* & 1.03 & 0.030 & -1.24 & M3IIIvar $\dagger$ & - & - \\
\hline HD 201381 & 0.73 & 0.032 & 1.00 & G8III & -0.15 & 2.8 \\
\hline HD 203504 & 0.86 & 0.030 & 0.71 & K1III & -0.14 & 1.2 \\
\hline HD 205390 & 0.41 & 0.030 & 6.30 & $\mathrm{~K} 2 \mathrm{~V} \dagger$ & - & - \\
\hline HD 206067 & 0.87 & 0.032 & 0.76 & K0III & -0.17 & 1 \\
\hline HD 206453 & 0.82 & 0.030 & -0.03 & G8III & -0.20 & - \\
\hline HD $206778 *$ & 1.78 & 0.030 & -4.19 & K2Ibvar & -0.05 & 6.5 \\
\hline HD 209747 & 1.06 & 0.030 & 0.32 & K4III & 0.00 & 2.3 \\
\hline HD $209750 *$ & 2.14 & 0.030 & -3.88 & $\mathrm{G} 2 \mathrm{Ib}$ & 0.18 & 6.7 \\
\hline HD $211931 *$ & 0.86 & 0.030 & 1.03 & $\mathrm{~A} 1 \mathrm{~V} \dagger$ & - & - \\
\hline HD 212943 & 0.78 & 0.030 & 1.33 & K0III & -0.33 & 0.6 \\
\hline HD 213042 & 0.44 & 0.030 & 6.71 & $\mathrm{~K} 4 \mathrm{~V} \dagger$ & - & - \\
\hline HD 2151 & 0.62 & 0.030 & 3.45 & G2IVvar & -0.18 & 3 \\
\hline HD $216032 *$ & 1.03 & 0.030 & -1.28 & $\mathrm{~K} 5 \mathrm{II} \dagger$ & - & - \\
\hline HD 217357 & 0.33 & 0.030 & 8.33 & $\mathrm{~K} 5-\mathrm{M} 0 \mathrm{~V} \dagger$ & - & - \\
\hline HD 21749 & 0.38 & 0.030 & 7.01 & $\mathrm{~K} 5 \mathrm{~V} \dagger$ & - & - \\
\hline HD 217580 & 0.45 & 0.036 & 6.34 & $\mathrm{~K} 4 \mathrm{~V} \dagger$ & - & 3.6 \\
\hline HD 218329 & 1.12 & 0.030 & -0.43 & $\mathrm{M} 2 \mathrm{III} \dagger$ & - & - \\
\hline HD 220339 & 0.42 & 0.030 & 6.35 & $\mathrm{~K} 2 \mathrm{~V} \dagger$ & - & 5.5 \\
\hline HD 220954 & 0.86 & 0.030 & 0.83 & K1III & -0.10 & 0.6 \\
\hline HD 27274 & 0.41 & 0.030 & 7.06 & $\mathrm{~K} 5 \mathrm{~V} \dagger$ & - & - \\
\hline HD 32450 & 0.33 & 0.030 & 8.66 & $\mathrm{MOV} \dagger$ & - & - \\
\hline HD 42581 & 0.30 & 0.030 & 9.34 & $\mathrm{M} 1-\mathrm{M} 2 \mathrm{~V} \dagger$ & - & 3 \\
\hline HD 4747 & 0.46 & 0.030 & 5.78 & $\mathrm{G} 8-\mathrm{K} 0 \mathrm{~V} \dagger$ & - & - \\
\hline HD $56855^{*}$ & 1.76 & 0.030 & -4.91 & $\mathrm{~K} 3 \mathrm{Ib} \dagger$ & - & - \\
\hline HD 59717* & 1.05 & 0.030 & -0.50 & $\mathrm{~K} 5 \mathrm{III} \dagger$ & - & - \\
\hline HD $68290 *$ & 0.84 & 0.030 & 0.95 & K0III & -0.03 & - \\
\hline HD $73840 *$ & 1.01 & 0.030 & -0.56 & K3III & -0.21 & - \\
\hline HD 74918* & 0.73 & 0.030 & 0.11 & G8III & -0.20 & - \\
\hline HD 75691 & 0.96 & 0.030 & -0.01 & K3III & -0.11 & - \\
\hline HD 78647 & 1.65 & 0.030 & -3.99 & K4Ib-IIvar & 0.23 & 8.9 \\
\hline HD 81101 & 0.82 & 0.030 & 0.62 & G6III $\dagger$ & - & - \\
\hline HD 82668 & 1.21 & 0.361 & -1.15 & $\mathrm{~K} 5 \mathrm{III} \dagger$ & - & - \\
\hline HD 85444 & 0.93 & 0.030 & -0.50 & G6-G8III & -0.14 & 2.9 \\
\hline HD 90432 & 1.04 & 0.030 & -1.15 & K4III & -0.12 & - \\
\hline HD 93813 & 0.96 & 0.030 & -0.03 & K0-K1II & -0.32 & - \\
\hline HD 95272 & 0.90 & 0.030 & 0.44 & K1III & -0.15 & - \\
\hline HD 9540 & 0.50 & 0.030 & 5.52 & $\mathrm{~K} 0 \mathrm{~V} \dagger$ & - & - \\
\hline HD 98430 & 0.89 & 0.030 & -0.31 & K0III & -0.40 & 1.8 \\
\hline
\end{tabular}



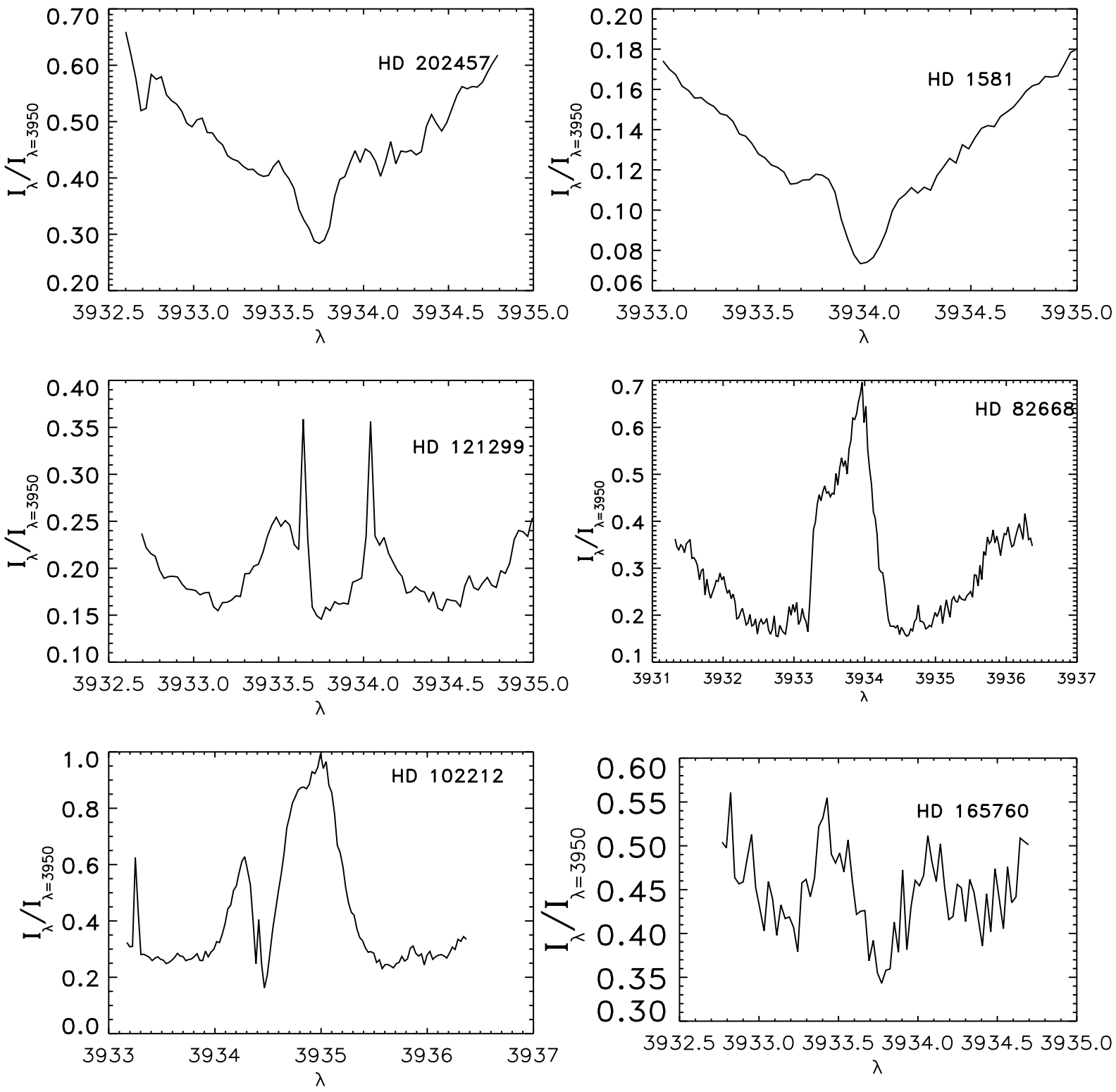

Fig. 1. Doubtful examples of CaII K line profiles. The spectra are affected by cosmic rays, may be blended with interstellar absorption, which strongly influence the emission profile observed in HD 82668, or, as in the case of HD 102212, show a blueshifted wind. In other cases, such as in HD 1581, the emission is very weak.

made to calibrate the WBR based on ground-based trigonometric parallaxes (Wilson 1959; Hodge \& Wallerstein 1966; Lutz \& Kelker 1975; Glẹbocki \& Stawikowski 1978). Wallerstein et al. (1999) (hereafter WMPG) used a very large sample of stars with Hipparcos parallaxes and relative measurement errors smaller than $20 \%$. As already stated, their work is based on the width measurements available in the literature, mostly from Wilson $(1967,1976)$, wich suffer from low spectral accuracy.

We note here that such measurements are based on the definition of $W$ given in Wilson \& Bappu (1957), corrected for instrumental broadening as in Wilson (1959) (see Sect. 3). So the quantity $W_{0}$ which they adopt is not exactly the same as ours, although the two quantities are expected to be strongly correlated.

WMPG used a linear least squares fitting both not weighted and weighted only in absolute magnitude with $\frac{\epsilon_{\pi}}{\pi}$. As WMPG advised, using weighted least squares means giving more weight to the lowest part of the diagram, containing the dwarfs that are, on the average, much closer, and therefore with smaller measurement errors on parallaxes. Using the weight for both the coordinates, as we did, does not produce to the same effect, because at the same time the dwarfs have also smaller $W_{0}$, and so larger relative measurement errors, i.e. larger standard errors for $\log W_{0}$. In Fig. 4 we show the comparison of our calibration and the weighted calibration of WMPG.

We think that this comparison is a strong test of the reliability of the WBR. In fact, with the only exception of having used Hipparcos parallaxes, the two samples are independent: less than the $40 \%$ of the stars we used in the computation are common to WMPG. Different stars means different distributions in magnitudes, metallicities and effective temperatures. Furthermore, different spectrographs were used, different 


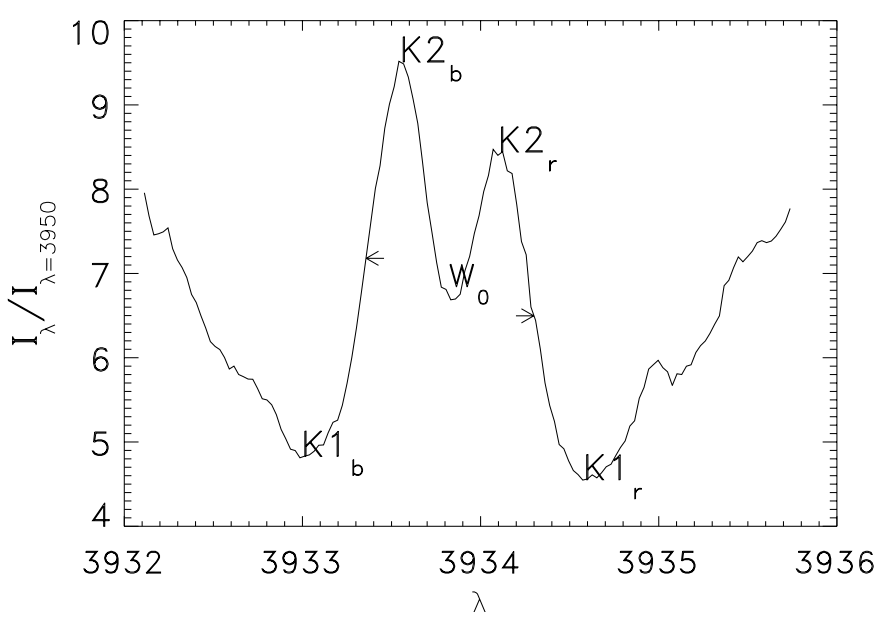

Fig. 2. Spectrum of HD 4128. Most of the spectra of our sample have a comparable quality.

Table 2. The resulting calibrations after three different sigma clipping criteria. $\sigma_{\mathrm{WBR}}$ is the the standard deviation of a single measurement. $\sigma_{a}$ and $\sigma_{b}$ are the standard deviations of the retrieved parameters, respectively, $a$ and $b$ of $M_{V}=a+b \cdot \log W_{0}$. The stability of the solution shows the reliability of the WBR as a distance indicator.

\begin{tabular}{cccccc}
\hline \hline criterion & $\begin{array}{c}\text { Number of stars } \\
\text { rejected }\end{array}$ & $\begin{array}{c}\text { Number of } \\
\text { iterations }\end{array}$ & \multicolumn{3}{c}{ Final result } \\
& 2 & 2 & 33.2 & -18.0 & 0.60 \\
\hline $3 \sigma$ & 5 & 3 & 33.5 & -18.3 & 0.56 \\
$2.5 \sigma$ & 16 & 9 & 33.6 & -18.3 & 0.49 \\
$2 \sigma$ & & \multicolumn{4}{c}{$\sigma_{a}=0.5 \& \sigma_{b}=0.3}$. \\
\hline
\end{tabular}

measurements (even based on different definitions of $W_{0}$ ) were performed as well as a different analysis. Taken at the face values, our calibration and WMPG's one are in good agreement for all stars brighter than $M_{V} \sim 2$, while for dwarfs, the discrepancy is stronger, reaching a difference of $1.5 \mathrm{mag}$ at $M_{V} \sim 9$.

In order to investigate how much of this discrepancy is due to the differences in $W_{0}$ measurements, we compared, for the 64 stars common to the two data sets, the $W_{0}$ measurements of WMPG and ours. Actually 20 of these stars are among the 33 not used in our WBR computation, but for the present comparison this is irrelevant. The two sets of measurements show, as expected, a very strong linear correlation: the slope of the $W_{0_{\text {our }}}$ vs. $W_{0_{\mathrm{WMPG}}}$ linear fitting is very much closed to unity: 1.003 , with an intercept of $-5.34 \mathrm{~km} \mathrm{~s}^{-1}$ (see Fig. 5).

If we subtract $5.34 \mathrm{~km} \mathrm{~s}^{-1}$ to our $W_{0}$ measurements, we obtain a data set homogeneous to that of WMPG, and performing the fitting with the new values gives following WBR: $M_{V}=29.7-16.3 \cdot \log W_{0}$. This result matches very well that of WMPG, as it can be seen from Fig. 4. We conlcude that the reliability of the WBR is excellent, and that the only reason for the discrepancy between the calibrations is the difference in the definition of $W_{0}$.

On the other hand we notice that care is needed in measuring $W_{0}$ : its definition, and possibly the resolution of the spectra used shoud be the same as those of the calibration adopted.

The fact that the difference between our and WMPG's measurements is about $5 \mathrm{~km} \mathrm{~s}^{-1}$, quite similar to the projected slit

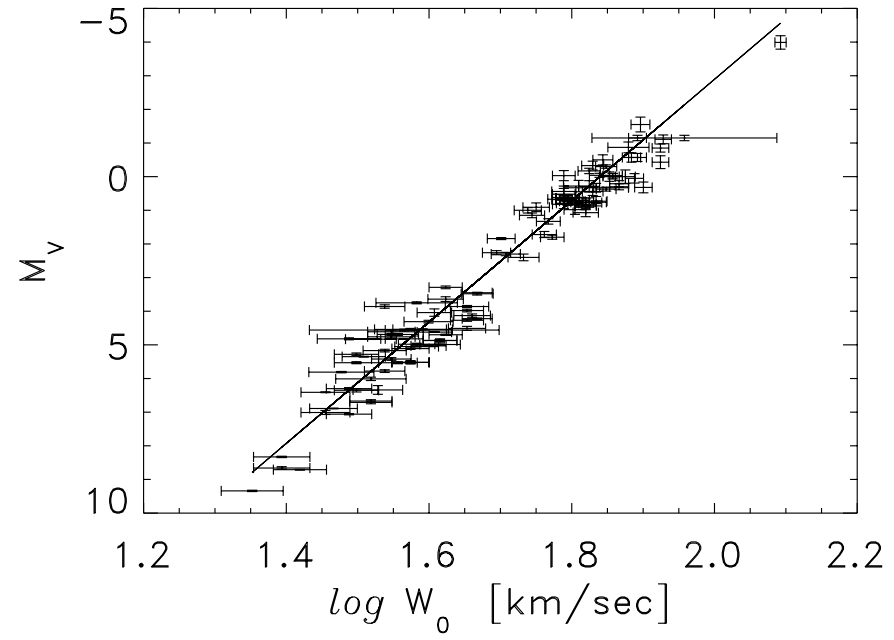

Fig. 3. Our calibration of the Wilson-Bappu Effect: $M_{V}=33.2-18.0$. $\log W_{0}$. This calibration is the $3 \sigma$ criterion one in Table 2 (HD 63077 and HD 211998 are not used). The error bars represent standard errors in both the coordinates.

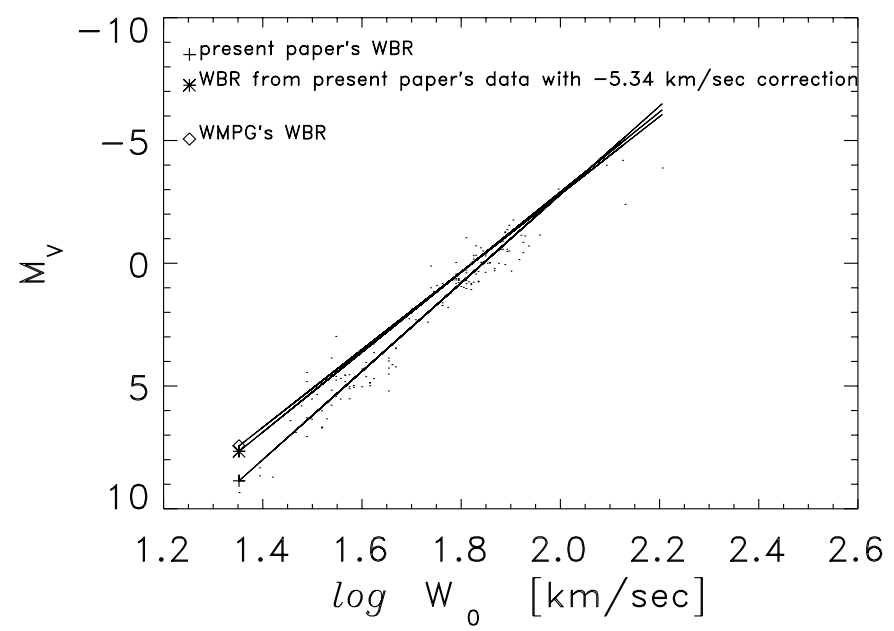

Fig. 4. Comparison between the following calibrations: the present paper's one $\left(M_{V}=33.2-18.0 \cdot \log W_{0}\right)$, the one we obtained after subtracting $5.34 \mathrm{~km} \mathrm{~s}^{-1}$ to all $W_{0}$ measurements and that of WMPG (the weighted one: $\left.M_{V}=28.83-15.82 \cdot \log W_{0}\right)$. The points refer to our data, including stars not used in the calibration because of the uncertainty in the parallax $\left(\frac{\sigma_{\pi}}{\pi}>0.1\right)$ or because they are binary or multiple systems.

width for $R=60000$, could suggest that the instrumental profile should indeed be linearly subtracted by our $W_{0}$ measurements to obtain an-instrument free calibration.

We do not believe that this is the case, because:

1. Other studies (Lutz 1970) have shown as the case of linear subtraction of instrumental profile is not the best choice;

2. Our observations of one star (HD 36069) taken at different resolutions to estimate this effect show that the variations observed in $W_{0}$ measurements are not better accounted for by a linear sibtraction of the instrumental profile, as shown from the measurements in Table 3.

For sake of completeness, we remind that the WBR is also valid for the $\mathrm{k}$-line of the $\mathrm{MgII}$, and that the best calibration to date is 


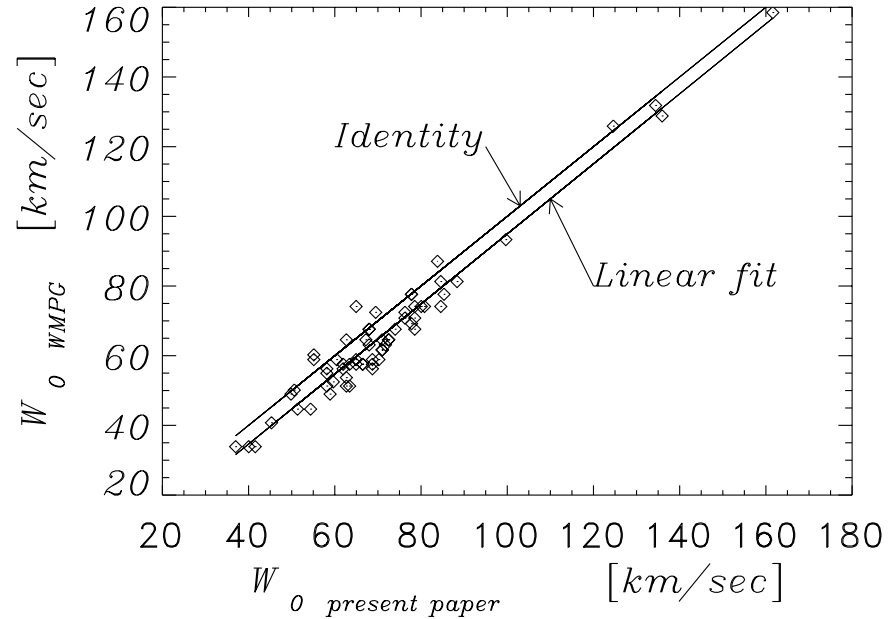

Fig. 5. Comparison between our measurements of $W_{0}$ and those used by WMPG.

the one of Cassatella et al. (2001), which also uses Hipparcos data and IUE spectra. Their result is: $M_{V}=34.56-16.75 \times$ $\log W_{0}$.

\section{Is the WBR a good distance indicator?}

The spread around the WBR is still too large to consider it as a reliable distance indicator for single stars. The question we are now going to investigate in this section is if the WBR is suitable to determine the distance of clusters of stars. A necessary condition which such clusters have to satisfy is, of course, that for a sufficient number of members, a high quality spectrum, showing a clear double reversal profile of the K-line, is available.

The possibility of using the WBR to determine accurate cluster distances is strictly related to the causes of the scatter: whether or not it is due to entirely random errors or systematic effects. Among the possible causes of scatter, we mention:

- random effects:

- measurement errors,

- cyclic variation in the chromospheric activity,

- variability of some of the stars of the sample;

- systematic effects:

- reddening,

- instrumental effects,

- Lutz-Kelker effect (hereafter LKE),

- hidden parameters, i.e. parameters other than $W_{0}$ and $M_{V}$ on which the WBR could depend.

White \& Livingston (1981) observed the chromospheric emission of the $\mathrm{K}$ line of the sun during a whole solar cycle. They found a maximum variation of $\log W_{0}$ of about 0.05 during such a period. If we assume that most of the stars are affected by a variation of $\log W_{0}$ of the same order of magnitude, the amount of scatter introduced by the cyclic variation of the chromospheric activity would represent a relevant fraction of the spread observed in the data. Nevertheless, this variability cannot fully explain the observed root mean square error of the
Table 3. Six $W_{0}$ measurements of HD 36079, made on spectra with different resolutions, both corrected and not corrected for instrumental broadening subtracting linearly the projected slit width. The variance in Col. 3 data is about 3 times that of Col. 1 and, most important, the corrected $W_{0}$ decreases with increasing correction. These measurements show that the linear subtraction of the instrumental profile is not appropriate for our data. A quadratic correction seems to be more justified for our data (see the last column).

\begin{tabular}{cccc}
\hline \hline Resolution & $W_{0}[\AA]$ & $W_{0_{\text {lincorr }}}[\AA]$ & $W_{0_{\text {quadcorr }}}[\AA]$ \\
\hline$R=110000$ & 0.923 & 0.887 & 0.922 \\
$R=80000$ & 0.915 & 0.866 & 0.914 \\
$R=60000$ & 0.925 & 0.859 & 0.923 \\
$R=60000$ & 0.918 & 0.852 & 0.916 \\
$R=40000$ & 0.929 & 0.831 & 0.924 \\
$R=30000$ & 0.953 & 0.822 & 0.944 \\
\hline Variance & $1.8 \times 10^{-4}$ & $5.6 \times 10^{-4}$ & $1.2 \times 10^{-4}$ \\
\hline
\end{tabular}

WBR fitting. With typical uncertainities in $W_{0}$ due to measurement errors and natural variations of the stellar line width of about $0.036 \AA$ (cf. Table 1), this error, for stars with intermediate widths, say $W_{0}=0.8 \AA$, accounts for about 0.35 mag of $\sigma_{\text {WBR }}=0.6 \mathrm{mag}$. Therefore, it is necessary to investigate further reasons of uncertainty in the determination of the WBR.

Among possible causes of biases we should consider reddening, the LKE Lutz \& Kelker (1973, hereafter LKP), instrumental effects and the presence of multiple systems.

\subsection{Lutz-Kelker effect}

The LKE is the bias due to the fact that a symmetric error interval $\left[\pi-\sigma_{\pi}, \pi+\sigma_{\pi}\right]$ around the estimated parallax $\pi$, does not correspond to a symmetric error interval in distances around $\frac{1}{\pi}$. The inner spherical corona centred in the Sun having radii $\frac{1}{\pi+\sigma_{\pi}}$ and $\frac{1}{\pi}$, has a volume smaller than the outer spherical corona. So, assuming a homogeneous space density for the stars, we expect that, for a fixed measured parallax, stars having a true distance greater than $\frac{1}{\pi}$, i.e. those in the outer corona, will outnumber the stars having a distance smaller than $\frac{1}{\pi}$. There is therefore a systematic trend to underestimate distances. The correction which has to be applied to each star, has been calculated in LKP. It depends only on the relative error $\frac{\sigma_{\pi}}{\pi}$.

Our sample has been selected to include only stars with $\left(\frac{\sigma_{\pi}}{\pi} \leq 0.1\right)$. Furthermore, out of the 119 stars, only 7 have $\frac{\sigma_{\pi}}{\pi}$ exceeding 0.075. For these values the LKE is negligible compared with other errors involved: $0.06 \mathrm{mag}$ for $\frac{\sigma_{\pi}}{\pi}=0.075$ and $0.11 \mathrm{mag}$ for $\frac{\sigma_{\pi}}{\pi}=0.1$ (See Table 1 in LKP).

\subsection{Reddening}

We have assumed that all stars have zero reddening. This assumption is justified by the fact that the sample stars are all within $\sim 200$ parsec.

The most distant star, HD 43455, has a distance of $205 \mathrm{pc}$, and it is the only one for which we were not able to find out a secure upper limit to the reddening. 
HD 78647 has a distance of 176 pc, and a galactic latitude lower than $7.6^{\circ}$, so we can get a rough estimation of its reddening on Neckel \& Klare's maps (Neckel et al. 1980). For this star, $A_{V}$ does not exceed $\sim 0.1$ mag.

The remaining stars are within 107 pc. According to Sfeir et al. (1999) (see their Fig. 2) the upper limit of the equivalent width of the D2 NaI line for such a distance is $200 \mathrm{~m} \AA$. From this quantity we can get the Hydrogen column density (Welsh et al. 1994): $N(\mathrm{HI}) \sim 2 \times 10^{20}$, which yields a colour excess: $E_{B-V} \sim 0.03$, or an upper limit for $A_{V}$ of about $0.1 \mathrm{mag}$.

Furthermore, 100 of the 119 stars in this sample, are within 75 parsec, so they are in the so called Local Bubble (see e.g. Sfeir et al. 1999), and they are not affected by detectable extinction.

\subsection{Multiple systems}

The presence of unrecognised multiple systems in the sample, gives rise to a systematic underestimation of $M_{V}(K)$, because we could associate the width of the $\mathrm{K}$ line emission of one component to the magnitude of the whole system. To avoid this effect, we checked all the objects of our sample on the SIMBAD database and we have excluded all the known multiple systems.

\subsection{Instrumental effects}

The measured $W_{0}$ is likely larger than the intrisic one because of the broadening introduced by the spectrograph. The larger the projected slit width is the stronger the intrumental broadening will be. We have shown in Sect. 4, by means of data in Table 3 , that a linear correction for instrumental broadening (i.e. subtracting the projected slit width from $W_{0}$ ) would not be appropriate. Similar results were found by Lutz (1970), who concluded that a quadratical correction should be used. To minimize this effect, our calibration is based on high resolution spectra (the projected slit width is about $0.066 \AA$ or $5 \mathrm{~km} \mathrm{~s}^{-1}$ ), and appling a quadratic correction even to the smallest $W_{0}$ value (that of HD 42581, $0.30 \AA$ ) we would obtain: $W_{0}-W_{0_{\text {corrected }}}=W_{0}-\sqrt{W_{0}^{2}-(0.066)^{2}}=0.0074 \AA$, well below its estimated measurement error, i.e. $\Delta W_{0}=0.03 \AA$. For larger values of $W_{0}, W_{0}-W_{0_{\text {corrected }}}$ is even smaller. Hence, the quadratical correction is negligible for all stars in our sample. We believe that the quadratical correction is more appropriate than the linear one, and it should be applyied when dealing with low resolution spectra, but it is not certain that such a small adjustment would represent a real improvement when dealing with data of resolution comparable to that used in this work.

\subsection{Other causes}

Many authors searched for additional parameters on which the WBR could depend, finding contradictory results. Glebocki \& Stawikowski (1978) proposed a corrected WBR with a term for the intensity of the emission that WMPG have rejected.

Parsons (2001), analysing the calibration of WMPG, suggested a trend for high luminosity stars that our data seem not to confirm: he suggested that $\mathrm{O}-\mathrm{C}$ (i.e. the difference between the

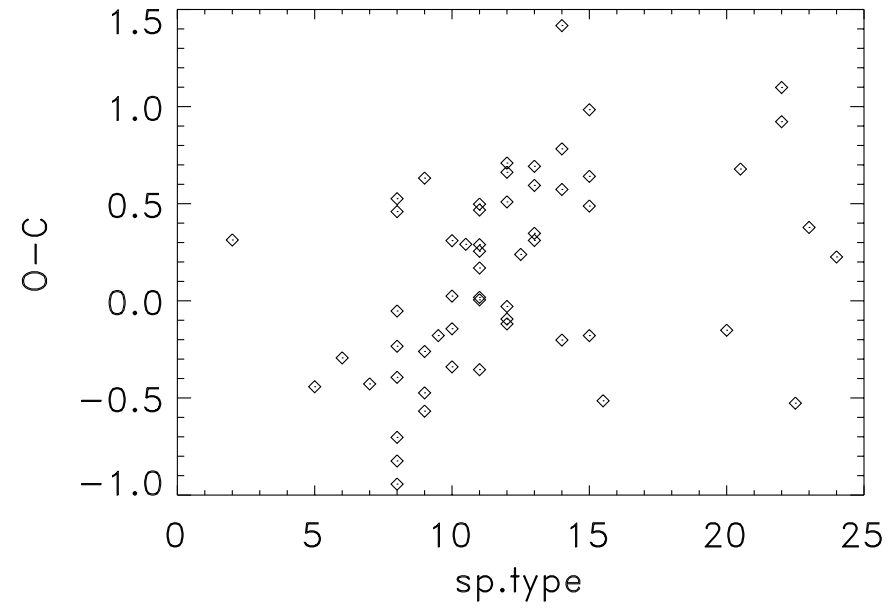

Fig. 6. The spectral type diagram vs. O-C. The spectral types are indexed as in Parsons (2001): 0 is for G0 stars, 1 for G1 and so on. Only luminous stars are plotted (no IV and V luminosity classes).

absolute magnitude from Hipparcos parallax and the one retrieved by means of the WBR) increases with increasing $T_{\text {eff }}$ for spectral types earlier than $\sim \mathrm{K} 3$, while the opposite is true for the other stars. He also concludes that this trend gets stronger for brighter stars. According to Fig. 6, while we can draw no conclusions for late type stars, our data seem to suggest a trend opposite to that proposed by Parsons (2001) for spectral types earlier than $\sim \mathrm{K} 3$.

The most obvious hidden parameter to search for is projected rotational velocity. High rotational velocity can influence $W_{0}$ in several ways, either because fast rotating stars will tend to be more active (see e.g. Cutispoto et al. 2002), or because the width of the line core may be modified by the higher rotational velocity (see e.g. Pasquini et al. 1989). We have 53 stars for which $V \cdot \sin i$ is available, and none are really fast rotators, only for one object $V \cdot \sin i$ exceeds $10 \mathrm{~km} \mathrm{~s}^{-1}$. Our conclusion is that, among slow rotators, there is hardly any dependence of the residuals on $V \cdot \sin i$ : we find a correlation coefficient of 0.12 .

We have finally searched for a dependence of the $\mathrm{O}-\mathrm{C}$ on metallicity. Such a dependence can also be expected, considering that in stars having lower abundances the core of the line may sample different layers of the atmosphere.

In particular we have checked whether the WBR is still valid for very metal poor stars. Figure 7 shows two O-C vs. $[\mathrm{Fe} / \mathrm{H}]$ diagrams: the one on the left refers to all the stars with available metallicities, in the other diagram only stars with $[\mathrm{Fe} / \mathrm{H}]<-0.3$ are plotted. A weak but not negligible dependence of the WBR on metallicity does exist, and it gets much stronger for metal poor stars. The correlation coefficient is 0.64 , and it becomes 0.82 when the 19 most metal poor stars are considered, as shown on the right panel of Fig. 7.

19 stars are too few to obtain any firm quantitative conclusions. In particular, the $\mathrm{O}-\mathrm{C}$ vs. $[\mathrm{Fe} / \mathrm{H}]$ relationship, to which they would point out (the straight line in right panel of Fig. 7), should be further investigated by means of a richer sample. The existence of such a relationship for metal poor stars has been 

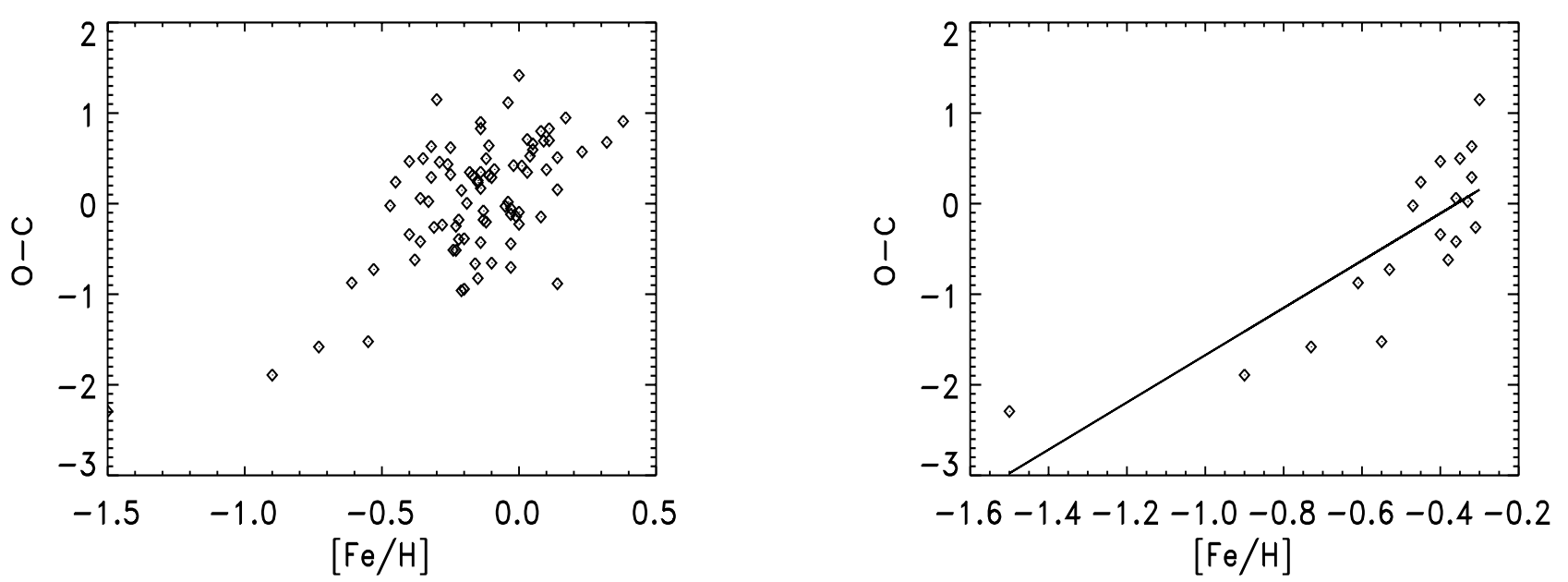

Fig. 7. The $\mathrm{O}-\mathrm{C}$ vs. $[\mathrm{Fe} / \mathrm{H}]$ diagrams both for all stars of the sample with available metallicities (on the left) and for metal poor stars only. On the latter is also shown the retrieved regression line, which has a slope as high as 2.61 .

independently suggested by Dupree \& Smith (1995), who studied 53 metal poor giants, none of which is in our sample.

We think that the WBR should be applied very carefully to very metal poor stars (e.g. stars more metal poor than $[\mathrm{Fe} / \mathrm{H}] \sim-0.4)$ and that further metal poor calibrators should be observed before applying it to very metal poor clusters.

\section{Application to M 67}

After deriving the WBR, and showing that the scatter is mostly due to random errors, we have the opportunity to test it on a group of stars belonging to a well studied open cluster. M $67 \mathrm{Ca}$ II spectra were published by Dupree et al. (1999) (see Figs. 2-4 therein) for 15 stars on the RGB and clump region, and they are suitable for our analysis of the WBR. Andrea Dupree kindly provided us with all the spectra in digital form.

Since M 67 has been extensively studied, the retrieved distance modulus can be compared with values obtained from other authors. Carraro et al. (1996) provide a detailed study of M 67. They derive, on the basis of the Colour Magnitude Diagram, $9.55 \leq(m-M)_{V} \leq 9.65 \mathrm{mag}$.

Montgomery et al. (1993) performed a photometric survey of the central region of M 67. They compared their photometry with two theoretical isochrones to retrieve distance modulus and age for M67. From $V, B-V$ CMDs, they found $(m-M)_{V}=9.60$ for both isochrones (but different ages were found), they have also used a $V, V-I \mathrm{CMD}$, giving $(m-M)_{V}=9.85$. Dinescu et al. (1995) found $9.7 \leq(m-$ $M)_{V} \leq 9.8 \mathrm{mag}$, obtained by letting $E_{B-V}$ varying between its upper (0.06 mag) and lower (0.03 mag) limits. Their isochrones were constructed using model atmospheres with new opacities. In Montgomery et al. (1993) other results from the literature are reported, ranging from 9.55 to 9.61 .

In summary, all distance modulus determinations for M 67 are in the range $9.55 \leq(m-M)_{V} \leq 9.85 \mathrm{mag}$.

M67 is a solar metallicity cluster, so we do not need to take care of the metallicity effect which may affect the WBR. The $W_{0}$ measurements were performed in the same way as for the calibration stars, and the results are given in Table 4. Out of the 15 stars of the original sample we have selected a subsample of 10, which suitable spectra were available, either for quality or clearness of the core reversal. In fact some of the spectra do not show a clear unambiguously recognisable double reversal feature, so that the measurement is unreliable. We did not use the stars with the following Sanders ID numbers (Sanders 1977): 258, 989, 1074, 1316, 1279. Even among the 10 selected stars some show a clearer profile than others, and for four of them the measurements were more uncertain (of the order of $0.1 \AA$ ) and they have been flagged with an asterisk in Table 4.

We have to consider that M67 spectra were acquired for other purposes, and in particular they have lower resolution and lower $S / N$ ratio than the typical calibration spectra, so we expect a standard error on the single measurement higher than the $\sigma_{\text {WBR }}$ derived above.

In Table 4 the distance modulus determinations for the single stars retrieved by means of the WBR are given. They range from 8.1 to $10.9 \mathrm{mag}$. The mean value is $\sim 9.7$. In spite of the poorer quality of the spectra, all the deviation can be explained on the base of the intrinsic spread around the WBR.

For sake of accuracy we have also taken into account the effect of the difference in resolution between the calibration spectra and the M 67 observations (5 and $11 \mathrm{~km} \mathrm{~s}^{-1}$ respectively). A simple, quadratic correction for the difference between the two projected slit widths is applied in the sixth column of Table 4 . The correction does not change the result in an appreciable way.

When considering all stars a simple mean gives $(M-m)=$ $9.62 \mathrm{mag}$; which becomes 9.65 when discarding the 4 most uncertain measurements.

We expect that the standard error in our determination of the distance of M 67 would be about: $\frac{\sigma_{\mathrm{WBR}}}{\sqrt{6}} \sim 0.3$ if we used 6 spectra of quality similar to those used for our calibration ( $\sigma_{\mathrm{M} 67}^{\prime} \sim 0.2$ if we had 10 spectra of the same quality).

Trying to push further this application would definitely represent a gross over interpretation of the data, however we find it extremely interesting and encouraging that a simple application, using published data, can provide a distance modulus 
Table 4. Data about the sample of the 10 stars in M67. Column 1: Sanders ID number (Sanders 1977) of the star. The stars with doubtful measurements are flagged with an asterisk. Column 2: Wilson-Bappu width in A. Column 3: apparent visual magnitude, from Table 1 in Dupree et al. (1999) (see references therein). Column 4: absolute magnitude inferred from the WBR. Column 5: retrieved distance modulus. Column 6: retrieved distance modulus using corrected widths: $W_{0_{\text {corrected }}}=\sqrt{W_{0}^{2}-P S W_{R=30000}^{2}+P S W_{R=60000}^{2}}(P S W$ is the projected slit width).

\begin{tabular}{cccccc}
\hline \hline Sanders ID & $W_{0}(\AA)$ & $m_{V}$ & $\begin{array}{c}M_{V}(\mathrm{~K}) \\
\text { no corr }\end{array}$ & $\begin{array}{c}(m-M)_{V} \\
\text { no corr }\end{array}$ & $\begin{array}{c}(m-M)_{V} \\
\text { corr }\end{array}$ \\
\hline S1010 & 0.851 & 10.48 & 0.657 & 9.823 & 9.742 \\
S1016* & 0.700 & 10.30 & 2.184 & 8.116 & 7.996 \\
S1074* & 0.784 & 10.59 & 1.298 & 9.292 & 9.196 \\
S1135 & 0.963 & 9.37 & -0.310 & 9.680 & 9.617 \\
S1221 & 0.854 & 10.76 & 0.629 & 10.131 & 10.050 \\
S1250* & 0.997 & 9.69 & -0.581 & 10.271 & 10.212 \\
S1479 & 0.868 & 10.55 & 0.502 & 10.048 & 9.970 \\
S1553 & 0.970 & 8.74 & -0.366 & 9.106 & 9.044 \\
S488 & 1.010 & 8.86 & -0.682 & 9.542 & 9.485 \\
S978* & 1.080 & 9.72 & -1.206 & 10.926 & 10.876 \\
\hline \multicolumn{6}{c}{ Mean value using all stars: } \\
Mean value using only unflagged stars: & 9.693 & 9.722 & 9.619 \\
\hline
\end{tabular}

in the range between 9.5 and 9.8, in excellent agreement with completely independent measurements, such as those obtained with main sequence model fitting.

\section{Conclusions}

We have shown that the coupling of CCD high resolution, high $S / N$ ratio data with the use of the Hipparcos parallaxes allows a good determination of the WBR. The root mean square error found around this relationship ( $0.6 \mathrm{mag}$ ) is not good enough to determine accurate distances to single stars, but it can be used to infer accurate distances of clusters or groups, provided that they are not too metal poor. This is possible because the uncertainties in the relationship are mostly due to random errors (measurements, cycles) and not from systematic effects. This implies that once one has observed a sufficient number of stars, $n$, the distance modulus standard error can be reduced to about $0.6 \mathrm{mag} / \sqrt{n}$. Its extension to metal poor objects (e.g. stars with $\mathrm{Fe} / \mathrm{H}<-0.4$ ) would require extra care to fully evaluate the impact of low metallicity on the relationship. When using our WBR in photometric parallax determinations, the resolution used should be comparable (within a factor $\sim 3$ ) to that of the calibration $(R=60000)$, to avoid large corrections, and care has to be exercised in measuring $W_{0}$, following the proper calibration definition.

Acknowledgements. We are greatly indebted to N. Bastian and P. Bristow for their careful reading of the manuscript. We thank the referee, Elena Schilbach, for very valuable comments and suggestions, which improved considerably the quality of this paper. Special thanks to A. Dupree, who kindly provided us with the M67 spectra.

\section{References}

Carraro, G., Girardi, L., Bressan, A., \& Chiosi, C. 1996, A\&A, 305, 849

Cassatella, A., Altamore, A., Badiali, M., \& Cardini, D. 2001, A\&A, 374,1085

Cayrel de Strobel, G., Soubiran, C., Friel, E. D., Ralite, N., \& Francois, P. 1997, A\&AS, 124, 229

Cutispoto, G., Pastori, L., Pasquini, L., et al. 2002, A\&A, 384, 491

Dinescu, D. I., Demarque, P., Guenther, D. B., \& Pinsonneault, M. H. 1995, AJ, 109, 2090

Dupree, A. K., \& Smith, G. H. 1995, AJ, 110, 405

Dupree, A. K., Whitney, B. A., \& Pasquini, L. 1999, ApJ, 520, 751

Hayes, D. S. 1985, IAU Symp., 111, 347

Glebocki, R., \& Stawikowski, A. 1978, A\&A, 68, 69

Glebocki, R., \& Stawikowski, A. 2000, Acta Astron., 50, 509

Hodge, P. W., \& Wallerstein, G. 1966, PASP, 78, 411

Lutz, T. E. 1970, AJ, 75, 1007

Lutz, T. E., \& Kelker, D. H. 1973, PASP, 85, 573

Lutz, T. E., \& Kelker, D. H. 1975, PASP, 87, 617

Montgomery, K. A., Marschall, L. A., \& Janes, K. A. 1993, AJ, 106, 181

Neckel, Th., Klare, G., \& Sarcander, M. 1980, A\&AS, 42, 251

Parsons, S. B. 2001, PASP, 113, 188

Pasquini, L., Pallavicini, R., \& Pakull, M. 1988, A\&A, 191, 253

Pasquini, L., Pallavicini, R., \& Dravins, D. 1989, A\&A, 213, 261

Pasquini, L. 1992, A\&A, 266, 347

Press, W. H., Teukolsky, S. A., Vetterling, V. T., \& Flanney, B. P. 1992, Numerical Recipies (Cambridge Univ. Press), 660

Sanders, W. L. 1977, A\&AS, 27, 89

Sfeir, D. M., Lallement, R., Crifo, F., \& Welsh, B. Y. 1999, A\&A, 346, 785

Wallerstein, G., Machado-Pelaez, L., \& Gonzalez, G. 1999, PASP, 111,335

Welsh, B. Y., Craig, N., Vedder, P. W., \& Vallerga, J. V. 1994, ApJ, 437, 638

White, O. R., \& Livingston, W. C. 1981, ApJ, 249, 798

Wilson, O. C., \& Bappu, M. K. V. 1957, ApJ, 125, 661

Wilson, O. C. 1959, ApJ, 130, 499

Wilson, O. C. 1967, PASP, 79, 46

Wilson, O. C. 1976, ApJ, 205, 823 